\title{
STAT3 $\alpha$ interacts with nuclear GSK3beta and cytoplasmic RISK pathway and stabilizes rhythm in the anoxic-reoxygenated embryonic heart
}

\author{
Sarah Pedretti $\cdot$ Eric Raddatz
}

Received: 12 October 2010/Revised: 23 December 2010/Accepted: 13 January 2011/Published online: 29 January 2011

(c) Springer-Verlag 2011

\begin{abstract}
Activation of the Janus Kinase 2/Signal Transducer and Activator of Transcription 3 (JAK2/ STAT3) pathway is known to play a key role in cardiogenesis and to afford cardioprotection against ischemiareperfusion in adult. However, involvement of JAK2/ STAT3 pathway and its interaction with other signaling pathways in developing heart transiently submitted to anoxia remains to be explored. Hearts isolated from 4-day-old chick embryos were submitted to anoxia $(30 \mathrm{~min})$ and reoxygenation $(80 \mathrm{~min})$ with or without the antioxidant MPG, the JAK2/STAT3 inhibitor AG490 or the PhosphoInositide-3-Kinase (PI3K)/Akt inhibitor LY-294002. Time course of phosphorylation of STAT3 $\alpha^{\text {tyrosine705 }}$ and Reperfusion Injury Salvage Kinase (RISK) proteins [PI3K, Akt, Glycogen Synthase Kinase 3beta (GSK3beta), Extracellular signal-Regulated Kinase 2 (ERK2)] was determined in homogenate and in enriched nuclear and cytoplasmic fractions of the ventricle. STAT3 DNA-binding was determined. The chrono-, dromo- and inotropic disturbances were also investigated by electrocardiogram and mechanical recordings. Phosphorylation of STAT3 $\alpha^{\mathrm{tyr} 705}$ was increased by reoxygenation, reduced $(\sim 50 \%)$ by MPG or AG490 but not affected by LY-294002. STAT3 and GSK3beta were detected both in nuclear and cytoplasmic fractions while PI3K, Akt and ERK2 were restricted to cytoplasm. Reoxygenation led to nuclear accumulation
\end{abstract}

Electronic supplementary material The online version of this article (doi:10.1007/s00395-011-0152-5) contains supplementary material, which is available to authorized users.

S. Pedretti $(\bowtie) \cdot$ E. Raddatz

Department of Physiology, Faculty of Biology and Medicine,

University of Lausanne, Rue du Bugnon 7,

1005 Lausanne, Switzerland

e-mail: sarah.pedretti@unil.ch of STAT3 but unexpectedly without DNA-binding. AG490 decreased the reoxygenation-induced phosphorylation of Akt and ERK2 and phosphorylation/inhibition of GSK3beta in the nucleus, exclusively. Inhibition of JAK2/ STAT3 delayed recovery of atrial rate, worsened variability of cardiac cycle length and prolonged arrhythmias as compared to control hearts. Thus, besides its nuclear translocation without transcriptional activity, oxyradicalsactivated STAT $3 \alpha$ can rapidly interact with RISK proteins present in nucleus and cytoplasm, without dual interaction, and reduce the anoxia-reoxygenation-induced arrhythmias in the embryonic heart.

Keywords JAK2/STAT3 pathway .

Anoxia-reoxygenation - Embryonic heart .

Oxyradicals · RISK pathway

\section{Introduction}

Although the embryo and the fetus develop normally in a relatively hypoxic environment [7, 31], cardiovascular function can be rapidly impaired by an accidental and transient intrauterine lack of oxygen [21] with possible long-term deleterious consequences. In the embryonic heart, the chrono-, dromo- and inotropic disturbances induced by anoxia-reoxygenation [39, 48] are associated with overproduction of Reactive Oxygen Species (ROS) and significant alterations of signaling pathways. In particular, the region-specific activation of Mitogen-Activated Protein Kinases (MAPKs) [12, 43] such as p38MAPK, extracellular signal-regulated kinase (ERK2) and c-jun $\mathrm{N}$-terminal kinase (JNK) and stimulation of protein kinase $\mathrm{C}$ and nitric oxide synthases [44] are part of the mechanisms involved in the response to anoxia-reoxygenation, 
with slight differences relative to the ischemic-reperfused adult heart [18]. In addition to these signaling pathways, the Janus Kinase 2/Signal Transducer and Activator of Transcription 3 (JAK2/STAT3) pathway is also activated by ischemia-reperfusion [1, 19]. JAK2 is a receptor-associated cytosolic protein which mediates signals to the nucleus by the subsequent phosphorylation/activation of STAT3 transcription factor. STAT3 can be phosphorylated on tyrosine ${ }^{705}$ by JAK upon activation and on serine ${ }^{727}$ mainly by ERK and p38MAPK [24]. It should also be noticed that expression of STAT3 is necessary for normal embryogenesis [53] and that JAK2/STAT3 activation is a prerequisite for the differentiation of embryonic stem cells into spontaneously beating cardiomyocytes [10]. Moreover, the JAK2/STAT3 pathway affords protection against reperfusion-induced injury in neonatal [51] and adult [19, 37] cardiomyocytes and can interact with other pathways including the Reperfusion Injury Salvage Kinase (RISK) pathway $[11,13,15,17,30]$. The RISK pathway is a group of pro-survival protein kinases including PhosphoInositide3-Kinase (PI3K), Akt/PKB, Glycogen Synthase Kinase 3beta (GSK3beta) and ERK1/2 which confers cardioprotection when activated at reperfusion but not in all species [50]. Although STAT3 is basically a transcription factor shuttling between cytoplasmic and nuclear compartments, the intracellular localization of its interactions with RISK pathway components as well as its role in cardiac function under pathological conditions remains to be explored. This work aimed (1) to establish the temporal profile of phosphorylation, the mechanisms of activation and the transcriptional activity of STAT3, (2) to assess the crosstalk between STAT3 and RISK pathway in the nuclear and the cytoplasmic compartments, and (3) to examine the role of activated STAT3 in the functional recovery of the anoxicreoxygenated embryonic heart.

\section{Materials and methods}

\section{Reagents}

Dimethylsulfoxide (DMSO) and antioxidant N2-mercaptopropionylglycine (MPG) were purchased from SigmaAldrich, JAK2/STAT3 inhibitor AG490 and PI3K/Akt inhibitor LY-294002 from Calbiochem and proteases inhibitors from Roche Biosciences. Rabbit antibody against phospho-Tyr ${ }^{705}$-STAT3 was from Ab Frontier. Antibodies against phospho-Ser ${ }^{727}$-STAT3, phospho-Tyr ${ }^{458}$-PI3K, PI3K, phospho-Ser ${ }^{43}$-Akt, Akt, phospho-Ser ${ }^{9}$-GSK3beta, GSK3beta, phospho-Ser ${ }^{641}$-GS, GS, phosphorylated ERK and ERK were from Cell Signaling Technology. Antibody against STAT3 was from Santa Cruz Biotechnology and the secondary antibody (goat anti-rabbit HRP conjugated) was from GE Healthcare. The enhanced chemiluminescence (ECL) western blot reagent kit was from PerkinElmer and films from GE Healthcare.

Preparation and in vitro mounting of the heart

Fertilized eggs from Lohman Brown hens were incubated during $96 \mathrm{~h}$ at $38^{\circ} \mathrm{C}$ and $90 \%$ relative humidity to obtain stage 24HH embryo (according to Hamburger and Hamilton [16]). The spontaneously beating hearts were carefully excised from explanted embryos by section at the level of the truncus arteriosus as well as between the sinus venosus and the atria. As previously described [45], the hearts were then placed in the culture compartment of a stainless steel chamber equipped with two windows for observation and maintained under controlled conditions on the thermostabilized stage $\left(37.5^{\circ} \mathrm{C}\right)$ of an inverted microscope (IMT2 Olympus, Tokyo, Japan). Briefly, the incubation compartment $(300 \mu \mathrm{L})$ was separated from the gas compartment by a $15 \mu \mathrm{m}$ transparent and gas-permeable silicone membrane (RTV 141, Rhône-Poulenc, Lyon, France). Thus, $\mathrm{PO}_{2}$ at the tissue level was strictly controlled and rapidly modified (within less than $5 \mathrm{~s}$ ) by flushing high-grade gas of selected composition through the gas compartment. The standard $\mathrm{HCO}_{3} / \mathrm{CO}_{2}$ buffered tyrode (supp. file) medium was equilibrated in the chamber with $2.31 \% \mathrm{CO}_{2}$ in air containing $19.5 \% \quad \mathrm{O}_{2}$ (normoxia and reoxygenation) or in $\mathrm{N}_{2}$ (anoxia) yielding a pH of 7.4. AG490 was reconstituted in DMSO. MPG, AG490 and LY-294002 were diluted in tyrode containing 0.5, 0.006 and $0.006 \%$ DMSO (vehicle), respectively, and present throughout the experimental protocol.

\section{Anoxia-reoxygenation protocol}

After a $30 \mathrm{~min}$ pretreatment at room temperature in vehicle, MPG (1 mM), AG490 $(10 \mu \mathrm{M})$ or LY-294002 $(10 \mu \mathrm{M})$, hearts were mounted in the chamber, stabilized 45 min under normoxia and submitted to anoxia (30 min) and reoxygenation $(80 \mathrm{~min})$. The hearts were harvested after stabilization (S), 10 (A10) and $30 \mathrm{~min}$ (A30) of anoxia and 10 (R10), 30 (R30), 40 (R40), 50 (R50), 60 (R60) and $80 \mathrm{~min}(\mathrm{R} 80)$ of reoxygenation. Control hearts were maintained under steady normoxia 60 and $90 \mathrm{~min}$ after S, corresponding to the time points R30 and R60, respectively. At the end of the experiment, the ventricles were carefully dissected on ice and stored at $-80^{\circ} \mathrm{C}$ for subsequent determinations.

\section{Protein homogenate}

For each sample, three ventricles were pooled because of the very small size of hearts. Ventricles were homogenized 
by sonication $3 \times 2 \mathrm{~s}$ in the ice-cold lysis buffer (supp. file) and protein content was measured by the method of Bradford (Coomassie protein assay kit, Pierce) with bovine serum albumin as standard.

\section{Immunoblotting}

Proteins from cellular extracts $(20 \mu \mathrm{g})$ were boiled with $1 / 3$ of SDS sample buffer (supp. file), separated on 10\% SDSpolyacrylamide gels $(1 \mathrm{~h}, 185 \mathrm{~V})$, and transferred to nitrocellulose membranes $(2 \mathrm{~h}, 100 \mathrm{~V})$. The equal loading in the membranes was systematically verified by performing Red Ponceau and by determining densitometry of total proteins and GAPDH. Membranes were probed with primary antibodies against phospho-Tyr ${ }^{705}$-STAT3 and phospho-GS (1:750); phospho-Ser ${ }^{727}$-STAT3, phosphoPI3K, STAT3 and PI3K (1:500); phospho-Akt, phosphoGSK3beta, phospho-ERK, Akt, GSK3beta, GS and ERK $(1: 1,000)$ diluted in $5 \%$ bovine serum albumin in tris-buffered saline tween (TBS-T, supp. file) (overnight, $4^{\circ} \mathrm{C}$ ). Blots were then incubated ( $1 \mathrm{~h}$, room temperature) with the secondary antibody $(1: 10,000)$ in $1 \%$ non-fat milk in TBS$\mathrm{T}$. Immunoreactive bands were detected using the ECL western blot reagent kit. Signal was semi-quantitatively analyzed using scanning densitometry (Quantity One software, Biorad). Bands of phosphorylated proteins were normalized to the total protein in the same sample and in the same membrane. More specifically for STAT3, the phospho- $\alpha$ isoform (P-STAT3 $\alpha$ ) was normalized to the total $\alpha$ isoform. Phosphorylation level at each time point of anoxia and reoxygenation was normalized to the respective preanoxic S level. We used total cell extracts from serumstarved HeLa cells prepared with IFN- $\alpha$ treatment (Cell Signaling Technology) as a positive control for P-Tyr STAT3, in which the $\alpha$ isoform was largely predominant relative to the beta isoform.

Enriched nuclear and cytoplasmic fractions preparation

Cytoplasmic and nuclear extracts were obtained as described elsewhere [27]. Twelve ventricles were homogenized in hypotonic buffer (supp. file). After addition of detergent Nonidet P-40 $(0,625 \%)$ and centrifugation, supernatants containing the cytoplasmic proteins were stored at $-80^{\circ} \mathrm{C}$. Pellets were resuspended in hypertonic buffer (supp. file), centrifugated and the resulting supernatants (nuclear fractions) were collected and stored at $-80^{\circ} \mathrm{C}$. Protein content was measured by the method of Bradford.

Electrophoretic mobility shift assay

A STAT3 oligonucleotide probe (supp. file) was labeled with $\alpha-{ }^{32} \mathrm{PdCTP}$ using the Klenow enzyme (Roche Applied
Science). $10 \mu \mathrm{g}$ of nuclear proteins were incubated with EMSA buffer (supp. file) and the probe for $20 \mathrm{~min}$ at room temperature. Samples were resolved on a nondenaturing polyacrylamide gel. Gels were transferred to Whatman $3 \mathrm{M}$ paper, dried under vacuum, and exposed to photographic films at $-80^{\circ} \mathrm{C}$ with intensifying screens. Densitometric analysis of autoradiographs was performed. A negative control was performed using either an antibody against STAT3 or an unlabeled probe. Ventricles isolated from hearts treated with $\mathrm{H}_{2} \mathrm{O}_{2}$ at $1 \mathrm{mM}$ for $1 \mathrm{~h}$ were used as a positive control for STAT3.

\section{Quantitative RT-PCR}

Twelve ventricles were homogenized in trizol (Invitrogen) and total RNA were purified by slight modifications of the method originally described by Chomczynski \& Sacchi [8]. The reverse transcription (RT) reaction was performed using the High capacity cDNA Reverse Transcription Kit and protocols from Applied Biosystem (ABI, Foster City, CA, USA). Briefly, the RT was run with $1.5 \mu \mathrm{g}$ of total RNA in a reaction volume of $20 \mu \mathrm{l}$ and aliquots of this reaction mixture (supp. file) were used for the subsequent PCR reactions. $5 \mathrm{ng}$ of cDNA was laid per well. Results are calculated using the $\Delta \mathrm{Ct}$ method [29].

\section{Recording of electrical and contractile activities}

Electrical and contractile activities were recorded simultaneously and continuously throughout in vitro experiments as previously described [45]. The PR and RR intervals, the QT duration, the ventricular apical shortening and the electromechanical delay (EMDv) were determined as previously described [45]. The maximal velocity of contraction and relaxation was calculated from the maximal positive and negative values of the first derivative of shortening and relaxation, respectively. Atrial rate was determined by measuring the delay between two $\mathrm{P}$ waves and the RR interval by the delay between peaks of two successive QRS complexes. We also assessed QRS widening, reflecting a possible reduction of ventricular conduction, by measuring the half-width of the QRS complex.

\section{Statistical analysis}

Because of the very small size of the heart (circa $60 \mu \mathrm{g}$ proteins) a total of about 2,300 chick embryos have been used in this study. Results are given as mean \pm standard error of the mean (SEM) for immunoblotting densitometry and as mean \pm standard deviation (SD) for functional parameters. The significance of any difference between two time points or two conditions was assessed using Mann-Whitney test. The statistical significance was defined by a value of $p \leq 0.05$. 


\section{Results}

Profile of STAT3 phosphorylation and STAT3 nuclear translocation during anoxia-reoxygenation

Only the $\alpha$ isoform of P-Tyr STAT3 was detectable under our conditions although both $\alpha(\sim 92 \mathrm{kDa})$ and beta ( $\sim 83 \mathrm{kDa}$ ) STAT3 isoforms were expressed in the ventricle (Fig. 1a). P-Tyr STAT3 $\alpha$ was not affected by anoxia (A10, A30) but increased during the first $60 \mathrm{~min}$ of reoxygenation (from R10 to R60) and returned to basal level at R80 (Fig. 1b). It should be noted that P-Tyr STAT3 $\alpha$ increase was more pronounced at R10 and R60. By contrast, P-Ser STAT3 $\alpha$ was not altered throughout reoxygenation compared to preanoxic level (Fig. 1b), the beta isoform bearing no serine phosphorylation site. The time-matched normoxic controls at S, R30 and R60 did not exhibit change in P-Tyr STAT3 $\alpha$ indicating that the culture conditions did not alter STAT3 activation relative to preanoxia (Fig. 1b). In the nuclear fraction P-Tyr STAT3 $\alpha$ increased at $\mathrm{R} 10$ and tend to increase at $\mathrm{R} 60(p=0.095)$ (Fig. 1c) whereas STAT3 was significantly increased
Fig. 1 Profile of STAT3 $\alpha$ activation in homogenates, nuclear and cytoplasmic fractions of the ventricle during anoxia-reoxygenation. a Representative immunoblots of the $\alpha$ isoform of tyrosine phosphorylated STAT3 (P-Tyr STAT $3 \alpha$ ), serine phosphorylated STAT3 (P-Ser STAT $3 \alpha$ ) and $\alpha$ and beta isoforms of STAT3 during anoxia (A10 and A30) and reoxygenation (R10 to $R 80)$ in homogenates. b Densitometric analysis of P-STAT $3 \alpha$ normalized to STAT3 $\alpha$. Black columns represent $\mathrm{P}-\mathrm{Tyr}$ STAT3 $\alpha$ and hatched columns P-Ser STAT3 $\alpha$ during anoxiareoxygenation and open columns P-Tyr STAT3 $\alpha$ in normoxic controls at matching time-points S, R30 and R60.

Representative immunoblots at $\mathrm{S}, \mathrm{R} 10, \mathrm{R} 60$ and R80 and densitometric analysis of P-Tyr STAT3 $\alpha$ (c) and STAT $3 \alpha$ and STAT3beta (d) in enriched nuclear fraction; P-Tyr STAT3 $\alpha$ (e) and $\operatorname{STAT} 3 \alpha$ and

STAT3beta (f) in cytoplasmic fraction. Data are expressed as fold change relative to the preanoxic value $(\mathrm{S}) .{ }^{*} p<0.05$, $* * p<0.01, * * * p<0.001$ versus $\mathrm{S}$. $N=4-9$ determinations for $\mathbf{b}$ and $N=4-13$ determinations for $\mathbf{c}-\mathbf{f}$

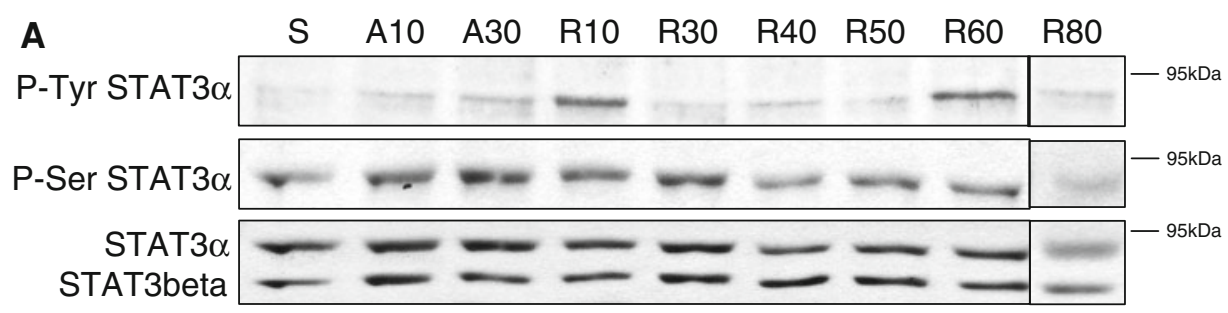

B
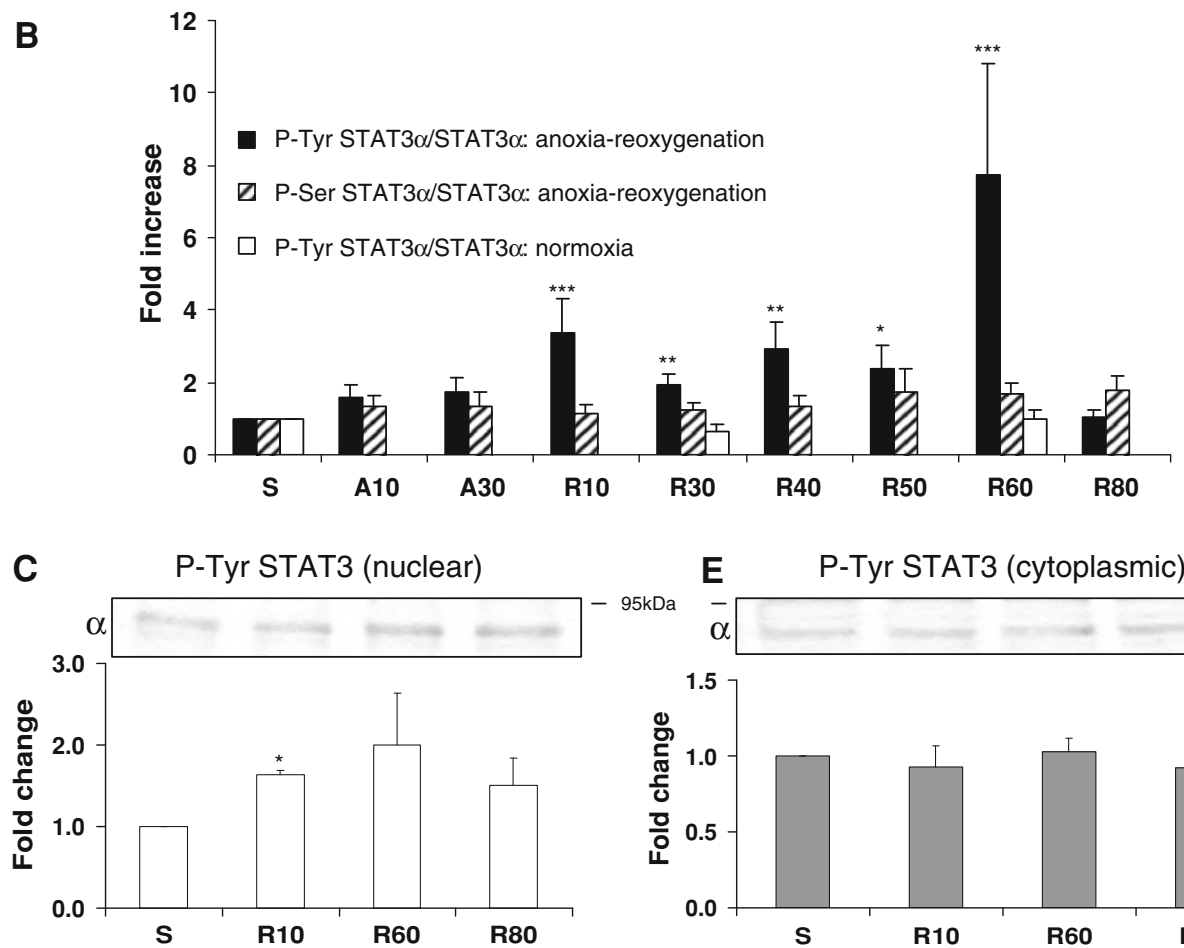

E P-Tyr STAT3 (cytoplasmic)

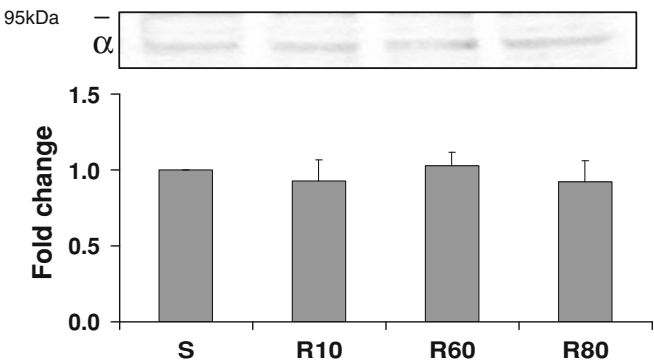

D

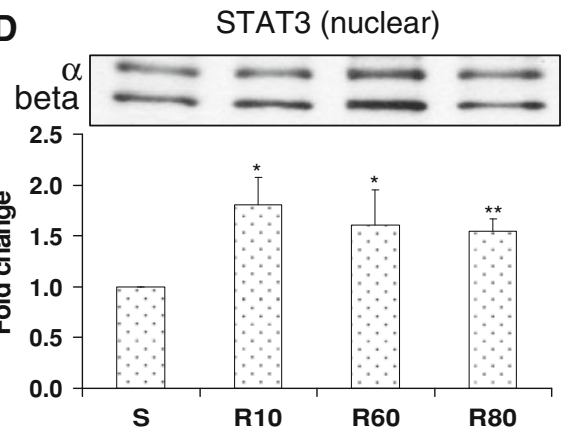

F $\quad$ STAT3 (cytoplasmic)

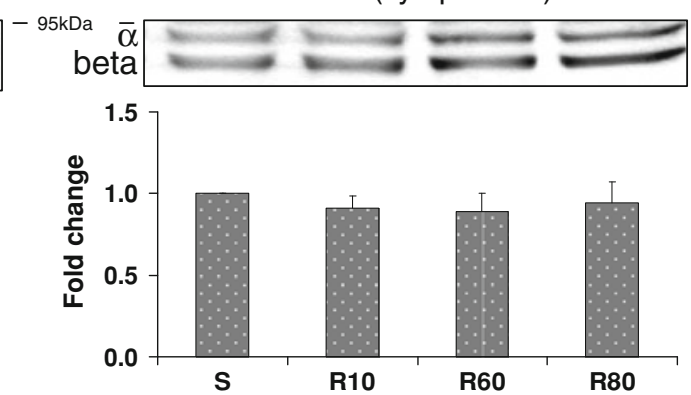


throughout reoxygenation (Fig. 1d). By contrast, in the cytoplasmic fraction P-Tyr STAT3 $\alpha$ and STAT3 were not affected (Fig. 1e, f). As total STAT3 increased in the nuclear fraction (Fig. 1d), P-Tyr STAT3 $\alpha$ obtained in both fractions was not corrected for total STAT3 in Fig. 1c, e.

Involvement of STAT3 in functional recovery

of the anoxic-reoxygenated embryonic heart

The recovery of atrial rate during reoxygenation was impaired and the coefficient of variability of the mean atrial rate was worsened by JAK2/STAT3 inhibition (Fig. 2a; Table 1). It should be noticed that during the first 15 min of reoxygenation the coefficient of variability of the mean atrial rate was increased in untreated hearts as well because of the unavoidable interferences of the reoxygenation-induced arrhythmias as described elsewhere [45]. However, at R30 the beat-to-beat variability of RR was clearly higher in AG490-treated hearts (Fig. 2b, c). All the other electrical and mechanical parameters were not significantly affected by AG490 (Table 1), i.e. atrioventricular (PR interval) and intraventricular conduction (QRS widening), QT duration and excitation-contraction coupling (EMDv). AG490 had no inotropic (shortening) or lusitropic (relaxation) effects. The types of arrhythmias (including atrial ectopy, atrioventricular block, Wenchebach) were similar in treated and untreated hearts. Furthermore, arrhythmias persisted throughout reoxygenation in $30 \%$ of
Fig. 2 STAT3 inhibition affected recovery of atrial rhythm during reoxygenation. a Mean atrial rate during anoxia-reoxygenation relative to the preanoxic level in vehicle (open triangles, $n=5$ ) or in $10 \mu \mathrm{M}$ AG490 (black diamonds, $n=9$ ), mean \pm SD. Note the greatest SD in treated hearts throughout reoxygenation. $* p<0.05$ versus vehicle. b Representative ECGs with P, $\mathrm{R}$ and $\mathrm{T}$ components in vehicle (V2, upper left panel) or in AG490 illustrating arrhythmias, i.e. PR prolongation followed by atrioventricular block (AVB; AG2, lower left panel) and variations of RR interval from one cardiac cycle to another at R30 (AG5, right panel).

c Distribution of individual RR intervals in five untreated hearts (vehicle, V1-V5) and in nine AG490-treated hearts (AG1AG9) at R30. The beat-to-beat analysis was performed on 100 successive cycles

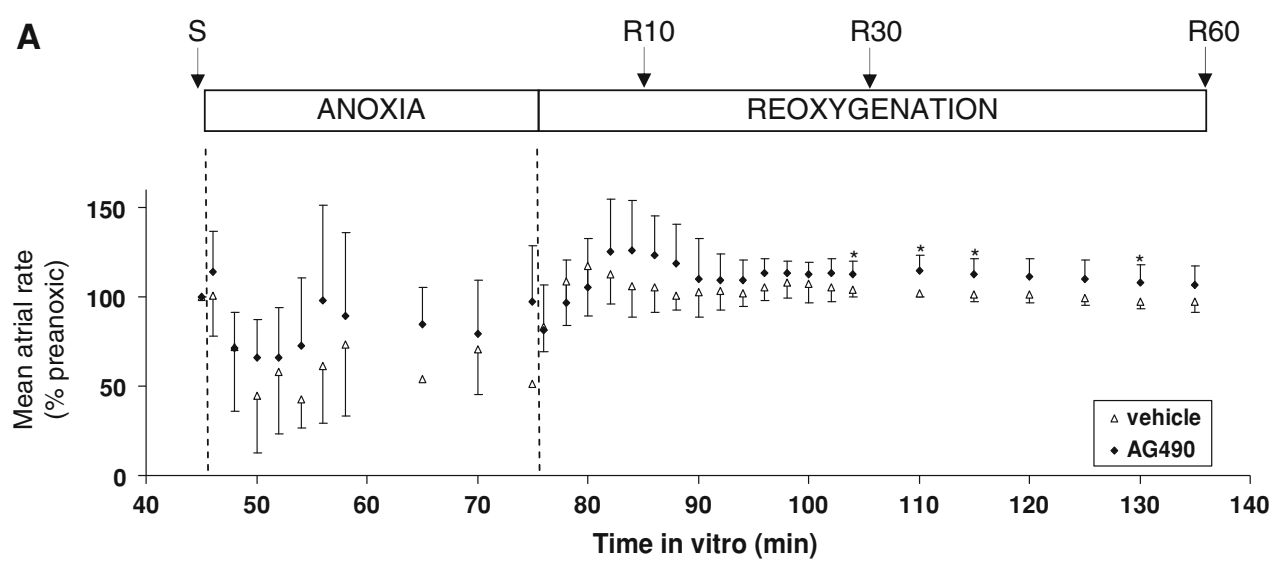

B
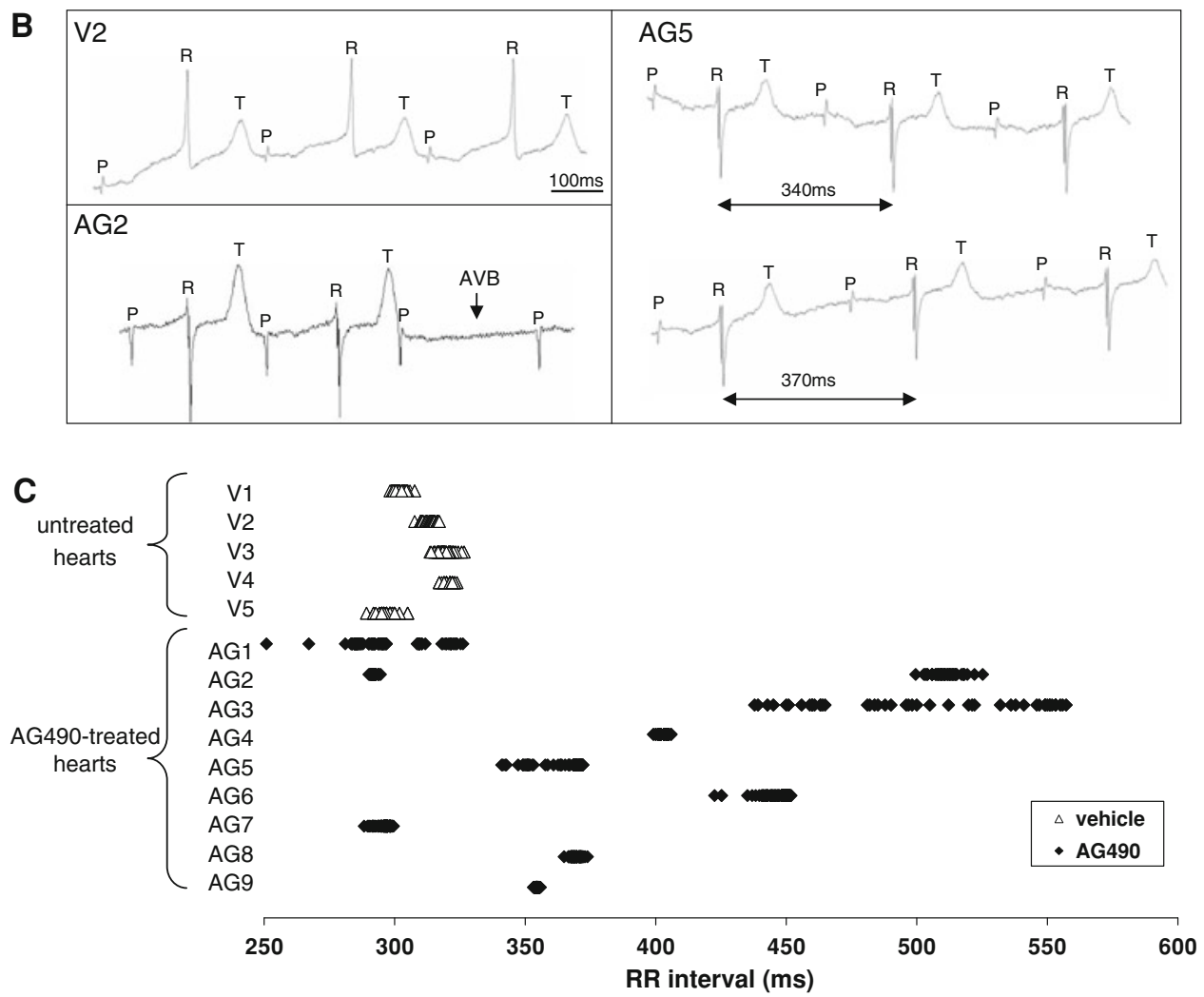
Table 1 Functional parameters under normoxia and during reoxygenation

\begin{tabular}{|c|c|c|c|c|}
\hline & $\mathrm{S}$ & R10 & $\mathrm{R} 30$ & R60 \\
\hline \multicolumn{5}{|c|}{ Mean atrial rate (bpm) } \\
\hline $\mathrm{v}$ & $191 \pm 8$ & $202 \pm 31$ & $198 \pm 8$ & $185 \pm 8$ \\
\hline AG & $160 \pm 26$ & $193 \pm 27$ & $180 \pm 33$ & $170 \pm 28$ \\
\hline \multicolumn{5}{|c|}{ Coefficient of variability of the mean atrial rate $(\%)$} \\
\hline $\mathrm{v}$ & 4 & 15 & 4 & 4 \\
\hline AG & 16 & 14 & 18 & 17 \\
\hline \multicolumn{5}{|c|}{ PR interval (ms) } \\
\hline $\mathrm{v}$ & $133 \pm 22$ & $169 \pm 34$ & $143 \pm 35$ & $126 \pm 32$ \\
\hline AG & $128 \pm 33$ & $145 \pm 25$ & $126 \pm 29$ & $119 \pm 33$ \\
\hline \multicolumn{5}{|c|}{ QT duration (ms) } \\
\hline $\mathrm{v}$ & $153 \pm 10$ & $182 \pm 5$ & $153 \pm 19$ & $150 \pm 26$ \\
\hline AG & $149 \pm 12$ & $137 \pm 9$ & $131 \pm 9$ & $139 \pm 11$ \\
\hline \multicolumn{5}{|c|}{ Contraction/relaxation velocity ratio } \\
\hline $\mathrm{v}$ & $0.96 \pm 0.26$ & $0.93 \pm 0.28$ & $1.00 \pm 0.14$ & $1.00 \pm 0.21$ \\
\hline AG & $1.05 \pm 0.12$ & $0.91 \pm 0.28$ & $1.03 \pm 0.30$ & $1.20 \pm 0.18$ \\
\hline \multicolumn{5}{|c|}{ Ventricular shortening $(\mu \mathrm{m})$} \\
\hline $\mathrm{v}$ & $16.5 \pm 9.6$ & $7.8 \pm 8.7$ & $18 \pm 15$ & $10.7 \pm 8.5$ \\
\hline AG & $14.1 \pm 7.9$ & $9.0 \pm 6.2$ & $9 \pm 8$ & $26 \pm 26$ \\
\hline \multicolumn{5}{|c|}{ EMDv (ms) } \\
\hline $\mathrm{v}$ & $26 \pm 10$ & $54 \pm 4$ & $33 \pm 1$ & $26 \pm 9$ \\
\hline AG & $25 \pm 4$ & $28 \pm 10$ & $25 \pm 3$ & $27 \pm 5$ \\
\hline \multicolumn{5}{|c|}{ QRS half-width (ms) } \\
\hline $\mathrm{v}$ & $3.4 \pm 1.5$ & $3.7 \pm 2.0$ & $3.2 \pm 0.8$ & $4.6 \pm 2.0$ \\
\hline AG & $3.0 \pm 1.3$ & $4.26 \pm 2.0$ & $3.5 \pm 1.6$ & $3.2 \pm 1.5$ \\
\hline
\end{tabular}

Electrical and mechanical parameters were not different in vehicle (V) and in $10 \mu \mathrm{M}$ AG490 (AG) at S, R10, R30 and R60. However, the interindividual coefficient of variability of the mean atrial rate appeared to be the highest in AG490-treated group at S, R30 and R60. Mean $\pm \mathrm{SD}$; Mann-Whitney test, $N=3-9$ determinations

$E M D v$ ventricular electromechanical delay the treated hearts, while they ceased at R30 in all other hearts.

ROS-dependent STAT3 $\alpha$ activation during post-anoxic reoxygenation

The level of P-Tyr STAT3 $\alpha$ was significantly decreased by MPG at R10 and R60, time-points at which activation of STAT3 was the strongest, with no effect at S (Fig. 3). The level of P-Tyr STAT3 $\alpha$ at R10 in Fig. 3 was lower than that shown in Fig. 1a, this effect was due to variability between experiments and/or the conditions of immunoblotting.

\section{STAT3 DNA-binding}

STAT3 DNA-binding activity determined by EMSA (Fig. 4a) did not vary during reoxygenation as illustrated by densitometry (Fig. 4b). Ventricles from hearts treated with $\mathrm{H}_{2} \mathrm{O}_{2}$ were used as a positive control to show that the technique was sensitive enough to detect any change in STAT3 DNA-binding activity in this embryonic tissue $\left(+33 \%\right.$ in hearts treated with $\mathrm{H}_{2} \mathrm{O}_{2}$ ) (Fig. 4c). A negative control of DNA-binding performed by adding an antibody against STAT3 or an unlabeled probe to the samples showed that the upper band corresponding to STAT3 disappeared under these two conditions (Fig. 4c). The unexpected finding that STAT3 was translocated without DNA-binding was supported by the fact that the level of mRNA expression of three STAT3 specific target genes in the context of ischemia-reperfusion, i.e. inducible NO synthase (iNOS), manganese superoxide dismutase (MnSOD) and cyclooxygenase- 2 (Cox-2), was not increased even at R80 (data not shown).
Fig. 3 ROS-dependent STAT $3 \alpha$ activation in the ventricle during post-anoxic reoxygenation. Immunoblots of P-Tyr STAT $3 \alpha$, STAT $3 \alpha$ and STAT3beta in vehicle $(\mathrm{V})$ or in $1 \mathrm{mM}$ MPG at S, R10 and R60 in homogenates (upper panels). Densitometric analysis of P-Tyr STAT $3 \alpha$ normalized to STAT3 $\alpha$ in $1 \mathrm{mM}$ MPG (black columns) and expressed as fold change relative to vehicle (open columns) at S, R10 and R60 in homogenates (lower panel).

$* * p<0.01$ vs vehicle.

$N=6-15$ determinations

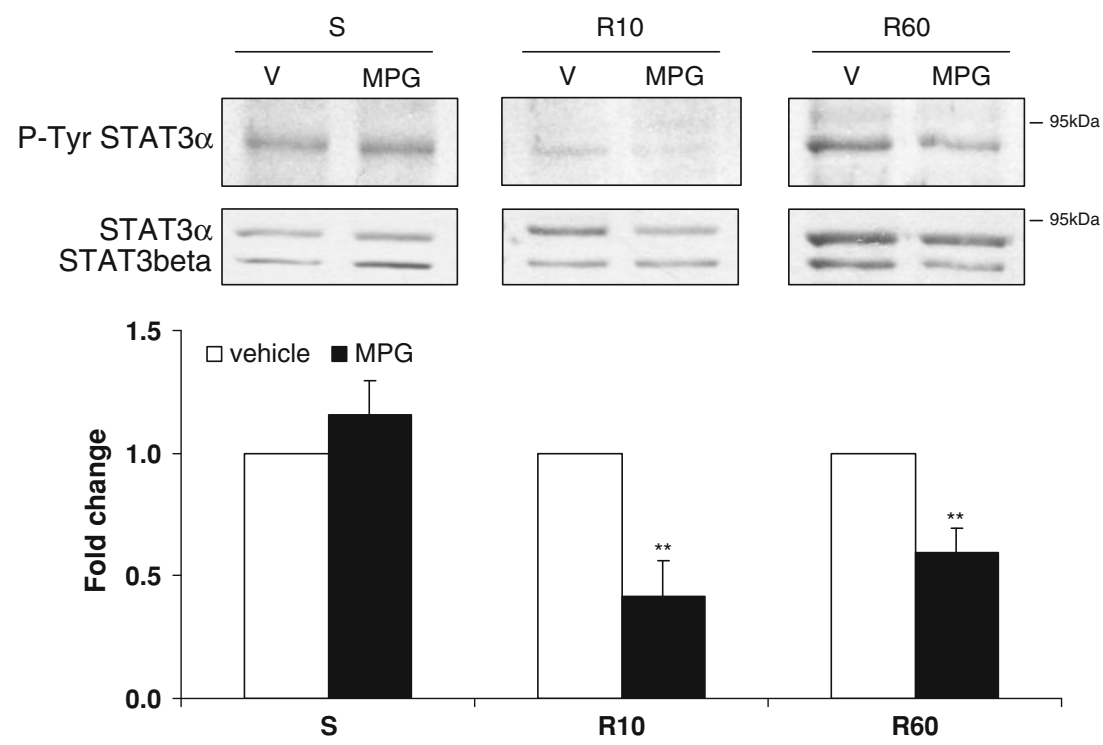


Fig. 4 At reoxygenation

STAT3 did not bind to DNA.

a Representative EMSA of

STAT3 at S, R10, R60 and R80

(duplicates) and $\mathbf{b}$ densitometric analysis of STAT3 in EMSA.

c On the left panel, positive control with ventricles isolated from hearts treated with $\mathrm{H}_{2} \mathrm{O}_{2}$ at $1 \mathrm{mM}$ for $1 \mathrm{~h}$ and on the right panel negative control with antibody $(\mathrm{Ab})$ against STAT3 and unlabeled probe at S, R10 and R60. $N=3-4$

determinations
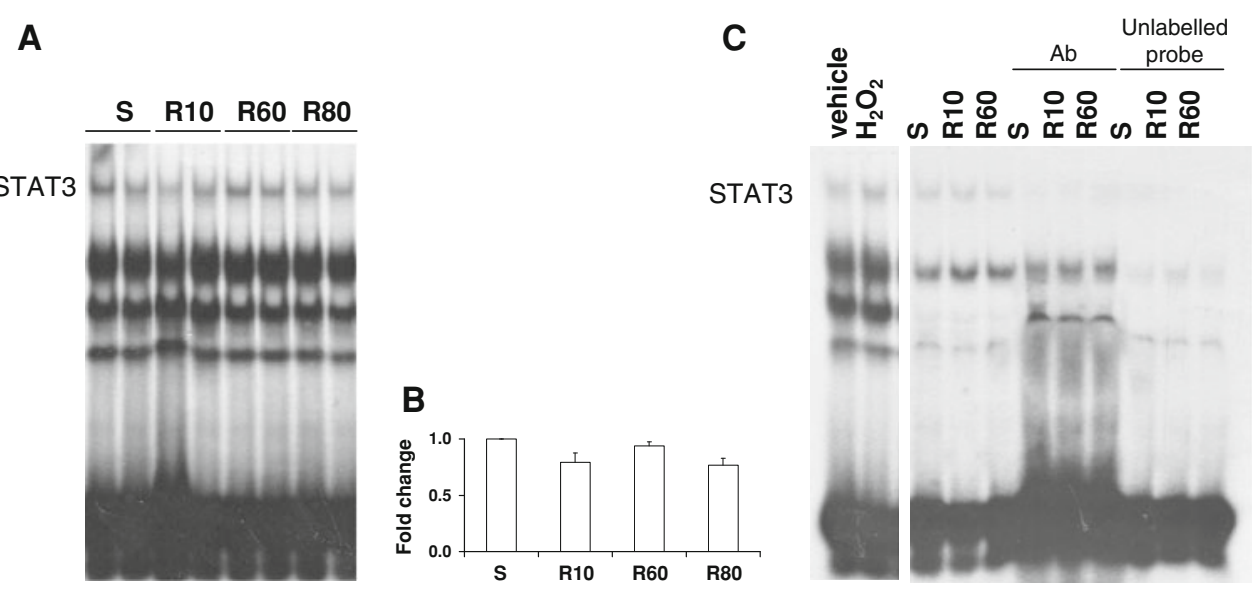

Distribution of PI3K, Akt, GSK3beta, glycogen synthase (GS) and ERK2 and possible crosstalk with STAT3

Using histone $\mathrm{H} 1$ and GAPDH as specific markers of enriched nuclear and cytoplasmic fractions, respectively, we found that phosphorylated and total forms of PI3K, Akt, GS and ERK2 were restricted to cytoplasm. Basal level of phosphorylated GSK3beta was markedly higher in the nuclear fraction than in the cytoplasmic fraction whereas the total form of GSK3beta was comparable in the two compartments (Fig. 5). The temporal pattern of phosphorylation varied from one component of the RISK pathway to another. PI3K and Akt phosphorylation peaked at R10 (Fig. 6a, b) while GSK3beta as well as GS phosphorylation was increased at R10 and R60 (Fig. 6c, d). Relative to the latest proteins, ERK2 phosphorylation was delayed at R60 (Fig. 6e). The possibility of interaction between RISK and JAK2/STAT3 pathways was assessed pharmacologically by AG490, a common JAK2/STAT3 pathway inhibitor. As expected, AG490 significantly decreased P-Tyr STAT3 $\alpha$ and P-Akt at R10 and R60 as well (Fig. 7a, c). AG490 reduced GS (Fig. 7d) and ERK2 (Fig. 7e) phosphorylation at R10 only with no effect on PI3K phosphorylation (Fig. 7b). GSK3beta being the only protein of the RISK pathway present in the nuclear compartment, together with STAT3, we checked separately the effect of STAT3 inhibition on the phosphorylation level of nuclear GSK3beta (Fig. 8). Nuclear and cytoplasmic P-GSK3beta significantly increased between S and R10 while GSK3beta did not vary (Fig. 8a). GSK3beta phosphorylation was decreased by AG490 in the nuclear fraction at R10 (Fig. 8b) but remained unchanged in the cytoplasmic fraction (Fig. 8c). The possible effect of PI3K/Akt on STAT3 phosphorylation was evaluated pharmacologically using LY-294002, a common PI3K/Akt pathway inhibitor. As expected, LY-294002 decreased P-Akt by 55\% at R10 (Fig. 9a) but did not affect P-Tyr STAT3 $\alpha$ (Fig. 9b).

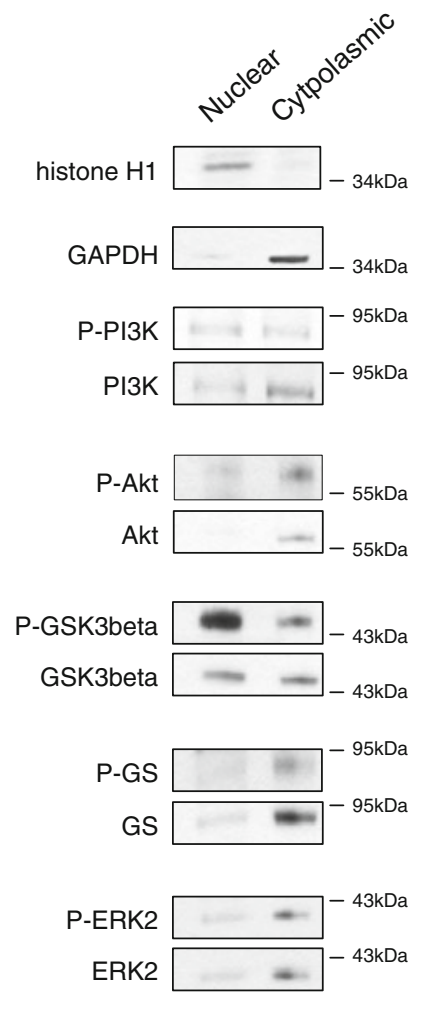

Fig. 5 Distribution and level of phosphorylation of PI3K, Akt, GSK3beta, GS and ERK2 in nuclear and cytoplasmic fractions of the ventricle. Representative immunoblots of basal content of phosphorylated and total forms of PI3K, Akt, GSK3beta, GS and ERK2 in enriched nuclear and cytoplasmic fractions of the ventricle. Histone $\mathrm{H} 1$ was used as a nuclear marker and GAPDH as a cytoplasmic marker (upper panels)

\section{Discussion}

To the best of our knowledge, this is the first time that the modulation of the JAK2/STAT3 pathway by a transient anoxic stress and its functional consequences are explored during early cardiogenesis. Our main findings indicate that (1) reoxygenation induces ROS-dependent phosphorylation 
Fig. 6 Profile of PI3K, Akt, GSK3beta, GS and ERK2 activation in the ventricle during post-anoxic reoxygenation. Representative immunoblots and densitometry of phosphorylated PI3K, Akt, GSK3beta, GS and ERK2 (a-e, respectively) normalized to total protein in homogenates at $\mathrm{S}$, R10 and R60. Data are expressed as fold increase relative to $\mathrm{S} . * p<0.05$, $* * p<0.01, * * * p<0.001$ vs $\mathrm{S}$ $N=5-12$ determinations
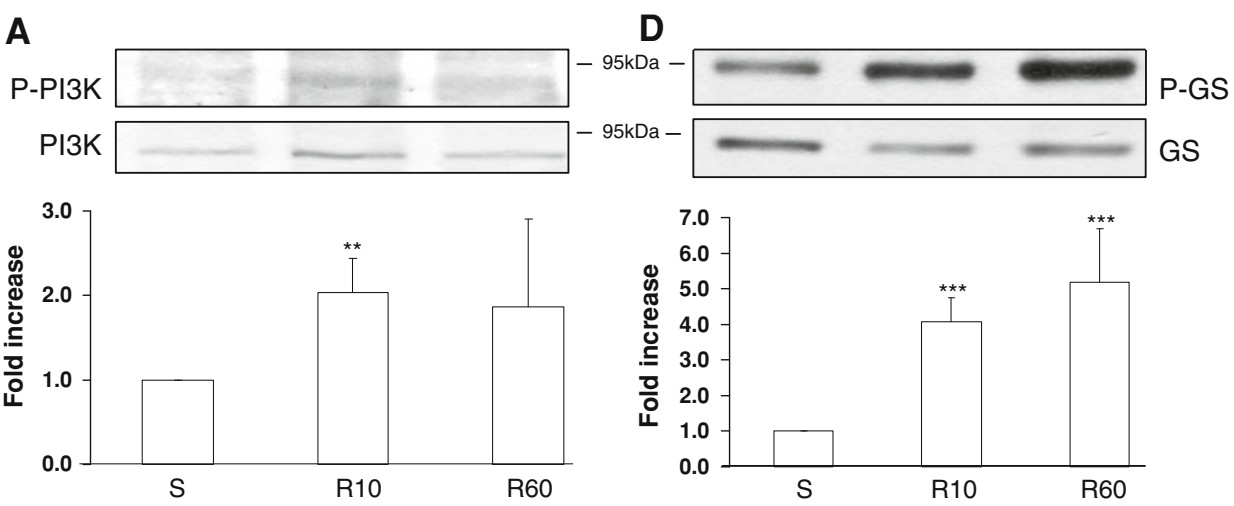

B

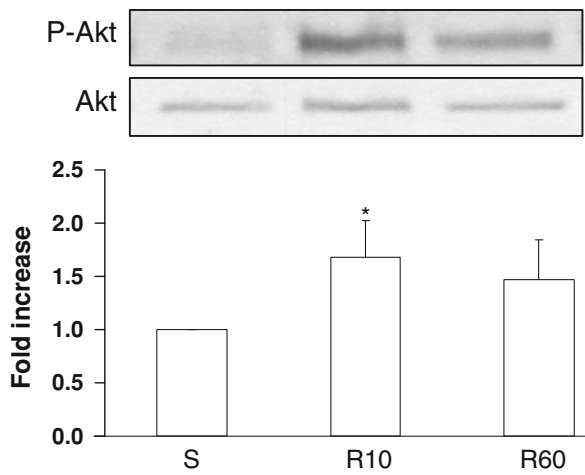

E
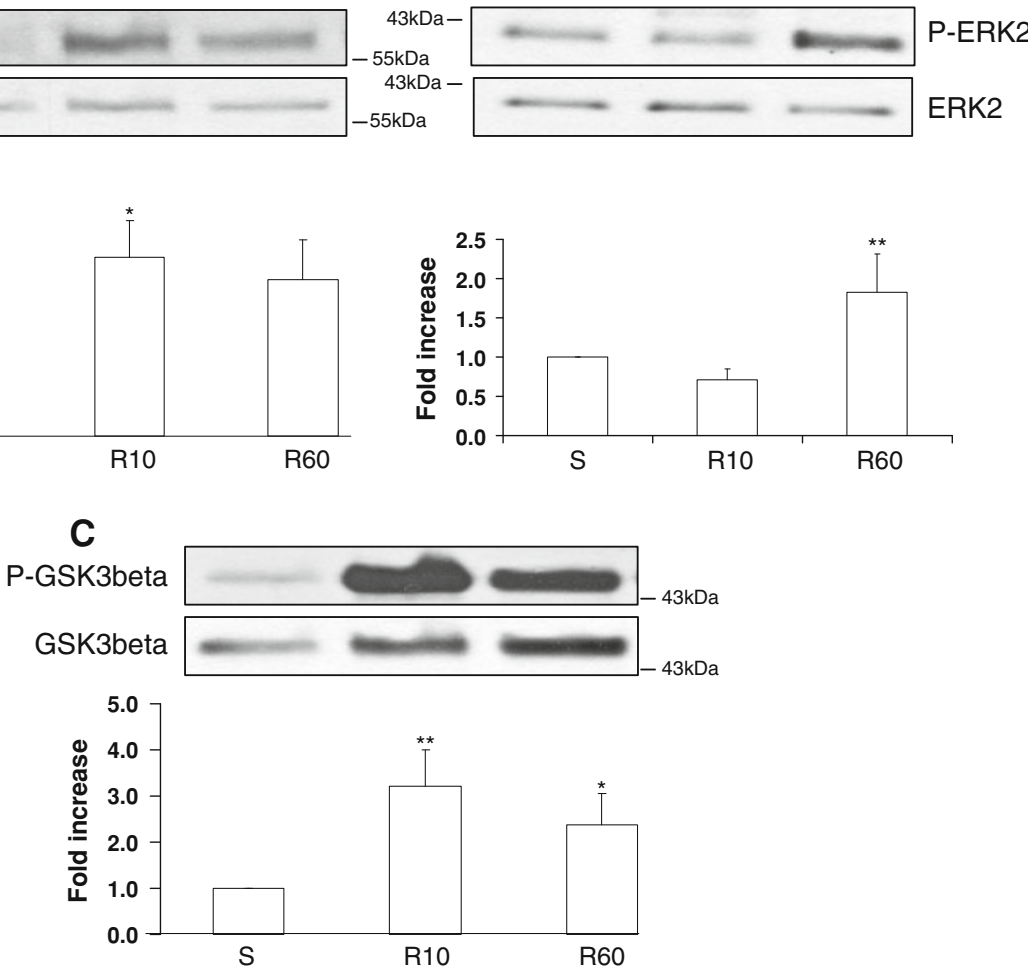

of Tyr-STAT3 $\alpha$ but not Ser-STAT3 $\alpha$, (2) P-Tyr STAT3 $\alpha$ translocates into the nuclear compartment without binding to DNA and inhibits GSK3beta, (3) phosphorylation of STAT $3 \alpha$ activates Akt and ERK2 and inhibits GS in the cytoplasmic compartment, (4) there is no dual interaction of STAT3 with proteins of the RISK pathway, and (5) activation of the JAK2/STAT3 pathway reduces the reoxygenation-induced arrhythmias.

\section{Characteristics and limitations of the model}

In the embryonic ventricle, only STAT3 and GSK3beta appeared to be present both in the nuclear and cytoplasmic fractions. PI3K, Akt, GS and ERK2 were restricted to the cytoplasm, whereas in neonatal and adult cardiomyocytes PI3K [42], Akt [35] and ERK [38] are detected also in the nuclear compartment. These observations suggest that the cytoplasm-nucleus shuttling of proteins, including transcription factors, may well depend on the level of differentiation and maturation of the cardiomyocytes. We were able to detect JAK2 by immunoblotting (not shown) but it was technically difficult to reveal its phosphorylated form with antibodies available on the market. AG490, a tyrosine kinase inhibitor which reduces JAK2 activity, significantly decreased the reoxygenation-induced STAT3 phosphorylation as expected, which validates this pharmacological approach to rapidly interfere with the JAK2/STAT3 pathway. Regarding ERK, as previously shown [12], only the p42 isoform (ERK2) is detectable in the embryonic and adult chicken heart, in contrast to neonatal and adult murine heart. As mentioned elsewhere [44], the isolated embryonic heart displays noticeable interindividual 
Fig. 7 JAK2/STAT3 inhibition significantly reduced reoxygenation-induced phosphorylation of Akt, GS and ERK2. On left panels, immunoblots of phosphorylated Tyr STAT3 $\alpha$, PI3K, Akt, GS and ERK2 (a-e, respectively) in vehicle (V) or in $10 \mu \mathrm{M}$ AG490 (AG) at S, R10 and R60 in homogenates. On right panels, densitometric analysis of phosphorylated Tyr STAT3 $\alpha$, PI3K, Akt, GS and ERK2 (a-e, respectively) normalized to total protein in $10 \mu \mathrm{M}$ AG490 (black columns) and expressed relative to vehicle (open columns) at $\mathrm{S}$, R10 and R60 in homogenates. $* p<0.05, * * p<0.01$, $* * * p<0.001$ vs vehicle. $N=4-11$ determinations
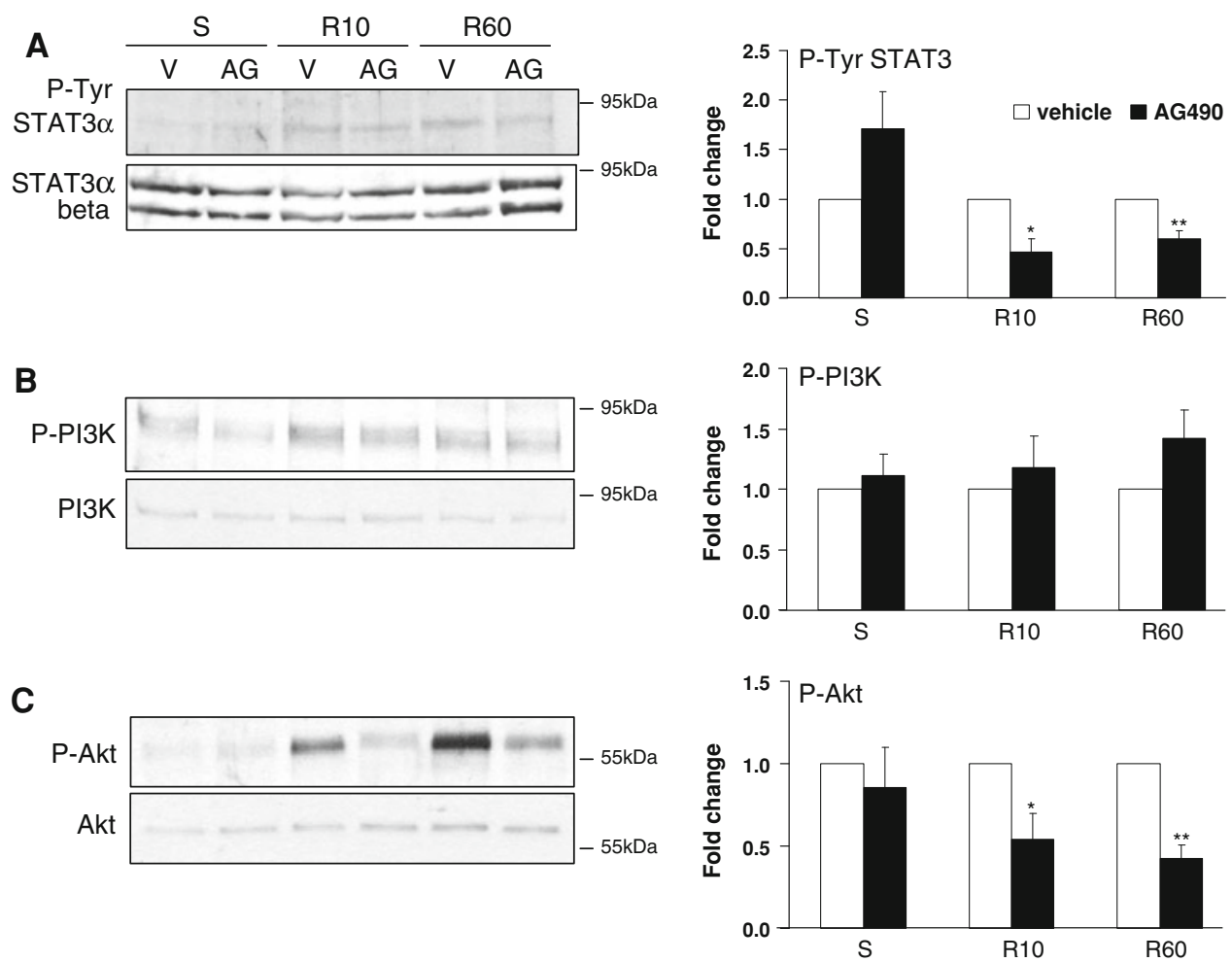

D
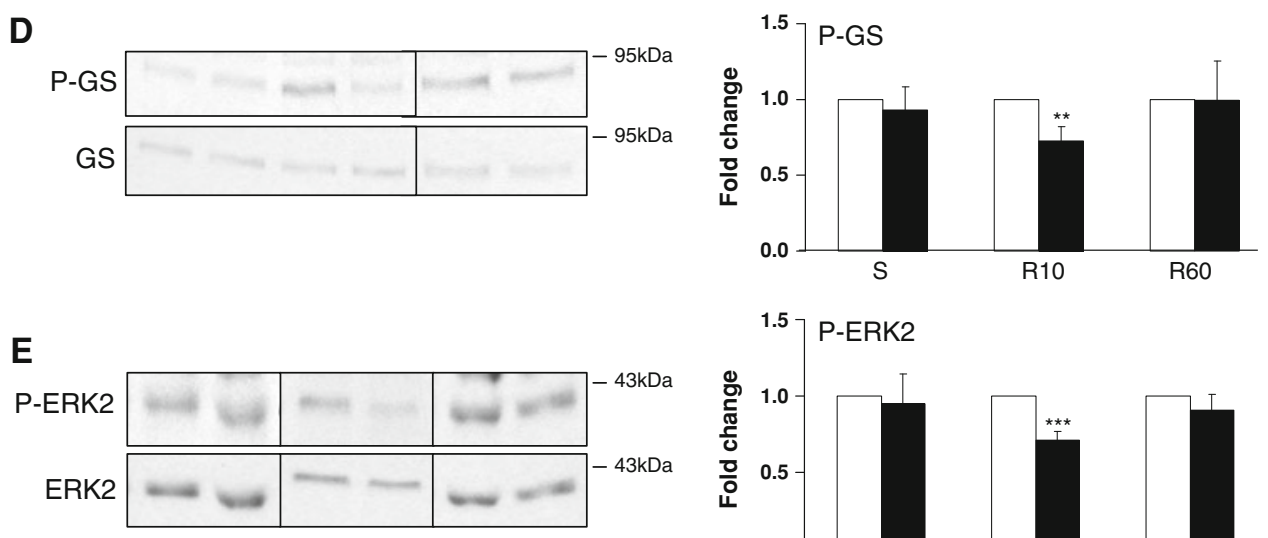

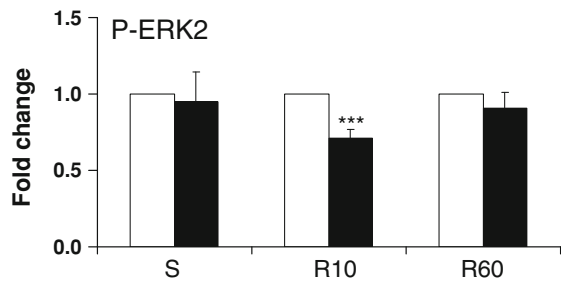

variations of the functional parameters and ECGs performed in similar conditions can also modestly differ in morphology from one experiment to another. Such variations could be due to slight interindividual differences in developmental stage, three-dimensional geometry of the hearts mounted in the chamber and variable vicinity of the recording electrodes. It is also conceivable that intrinsic oscillations of activation of signaling pathways in the embryonic cardiomyocytes [23], combined with variable rate of proliferation and differentiation in the different cell populations, could partly contribute to increase the interindividual variations of STAT3 phosphorylation determined at a given time point (specially at R60). A significant variability of the phosphorylation level of signaling proteins such as JNK, p38 and ERK2, has also been observed in the same experimental setting [12, 43]. It should be noticed that, contrary to the adult, the ventricle of the 4-day old embryonic chick heart is mostly composed of proliferating and differentiating cardiomyocytes with few endothelial and epicardial cells and no fibroblasts [49].

STAT3 phosphorylation and translocation during anoxia-reoxygenation

The full-length STAT, the $\alpha$ isoform, can undergo alternative splicing at the $3^{\prime}$ end gene transcripts leading to shorter beta isoform with truncated C-terminal domain (lacking 48 amino acids) [28]. STAT3 $\alpha$ and STAT3beta are distinctly different in their activation, transcriptional activities, and biological functions [47]. Our data indicate 
Fig. 8 Reoxygenation-induced phosphorylation/inhibition of GSK3beta in the nuclear compartment depended on JAK2/STAT3 activation. a Representative immunoblots of P-GSK3beta and GSK3beta in enriched nuclear and cytoplasmic fractions in vehicle $(V)$ or in $10 \mu \mathrm{M}$ AG490 $(A G)$ at $\mathrm{S}$ and R10. Histone H1 was used as a nuclear marker and GAPDH as a cytoplasmic marker. b Representative immunoblots (upper panels) and densitometric analysis (lower panels) of phosphorylated GSK3beta in enriched nuclear fraction and $\mathbf{c}$ in cytoplasmic fraction in AG490 (AG, black columns) and expressed as fold change relative to vehicle $(\mathrm{V}$, open columns) at S, R10 and R60. $* * * p<0.001$ versus vehicle. $N=3-6$ determinations

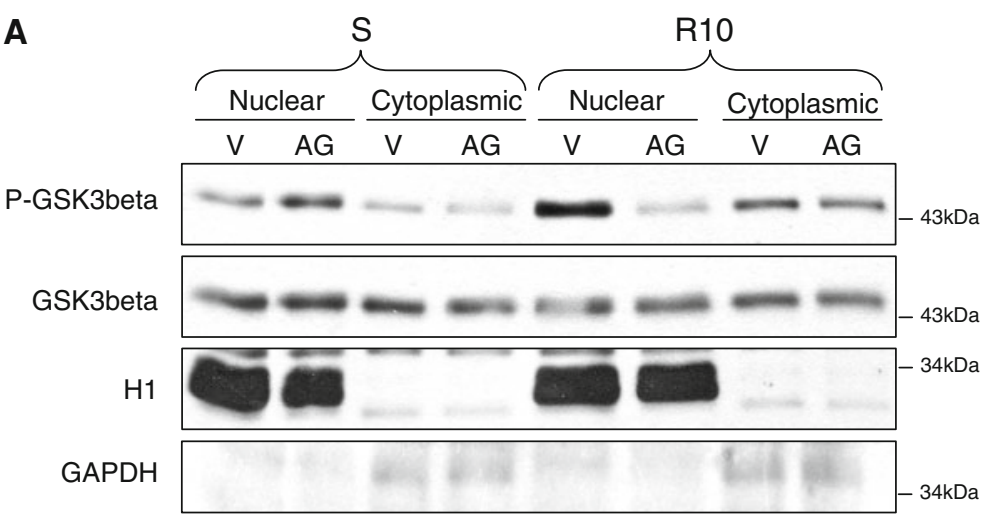

B

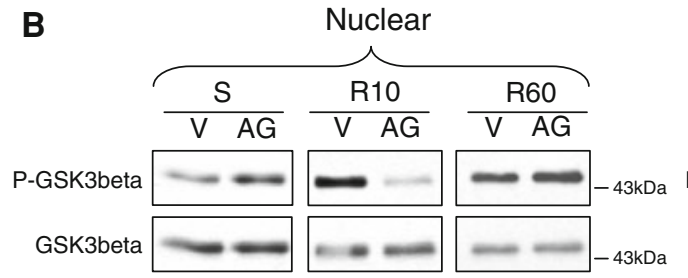

C
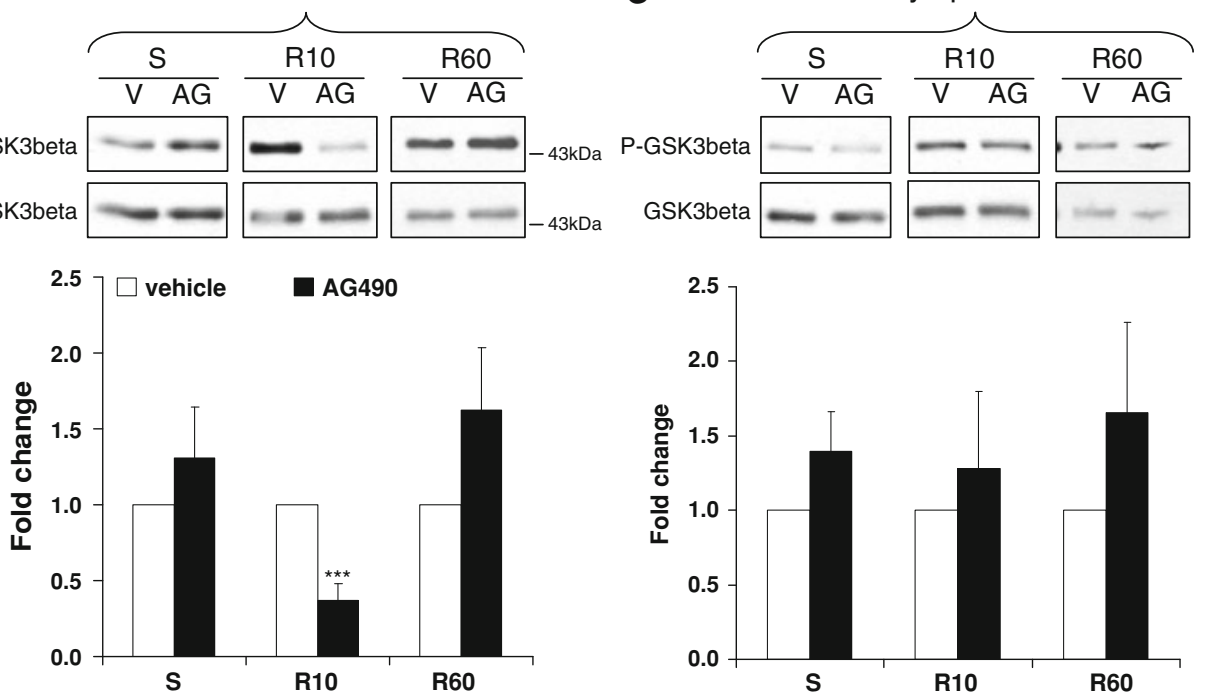

A
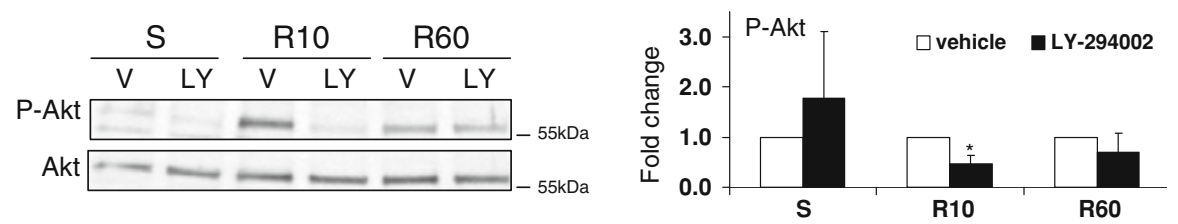

B

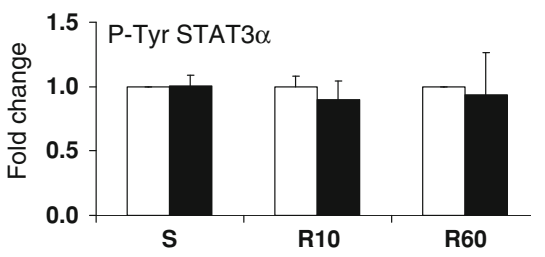

Fig. 9 PI3K/Akt inhibition by LY-294002 did not affect reoxygenation-induced phosphorylation of STAT3. On left panels, immunoblots of phosphorylated and total forms of a Akt and b STAT3 in vehicle (V) or in $10 \mu \mathrm{M} \mathrm{LY-294002} \mathrm{(LY)} \mathrm{at} \mathrm{S,} \mathrm{R10} \mathrm{and} \mathrm{R60} \mathrm{in}$ homogenates. On right panels, densitometric analysis of

that $\alpha$ and beta isoforms of STAT3 are strongly expressed in the embryonic heart but that only the $\alpha$ isoform is responsive to post-anoxic reoxygenation whereas phosphorylated a Akt and $\mathbf{b}$ Tyr STAT3 $\alpha$ normalized to total protein in $10 \mu \mathrm{M}$ LY-294002 (black columns) and expressed relative to vehicle (open columns) at S, R10 and R60 in homogenates. $* p<0.05$ versus vehicle. $N=3$ determinations

STAT3beta is known to have a critical developmental function [9] which was not investigated in this work. As tyrosine rather than serine site was phosphorylated by 
reoxygenation, the possibility of STAT3 activation by MAPKs [24] can be ruled out under our conditions since serine is preferentially phosphorylated by these pathways. More specifically, although p38MAPK and ERK2 are known to be activated at R10 and R30, respectively [12], they did not phosphorylate STAT3 ${ }^{\text {Ser727 }}$. At R10 activation of STAT3 observed in homogenate principally reflected what took place in the nuclear compartment since only nuclear P-Tyr STAT3 $\alpha$ increased significantly as in pharmacological postconditioned adult murine hearts [26]. At R60 nuclear P-Tyr STAT3 $\alpha$ tended to remain higher than the preanoxic level and the rise in nuclear STAT3 observed at R10 persisted throughout reoxygenation suggesting that translocated STAT3 was sequestered in the nuclear compartment. An increase in nuclear phosphorylated STAT3 is observed also in the ethanolamine-induced protection of the adult heart against ischemia-reperfusion injury [22].

The presence of mitochondrial STAT3 in our preparation cannot be ruled out since STAT3 is known to be present in mitochondria of several tissues [2, 14, 55] and it has also recently been shown that mitochondrial activated STAT3 contributes to cardioprotection by stimulation of respiration and inhibition of mPTP opening [4]. However, in embryonic myocardium mitochondria are scarce and not fully differentiated by contrast with adult tissue and their contribution to the cellular content of STAT3, if any, should be minor.

Involvement of the JAK2/STAT3 pathway

in cardiac rhythm

We have previously shown that the embryonic heart fully recovers at R60 [44] but the signaling pathways underlying the mechanisms of recovery remain relatively unexplored. Activated STAT3 is known to exert its late cardioprotective action (e.g., antiapoptotic properties [30]) mainly via alteration of transcription of target genes principally induced by pre- or postconditioning [3]. However, the short-term consequences of STAT3 activation on the electrical and mechanical activities have never been investigated, including those in the developing heart. At R30, inhibition of JAK2/STAT3 gave rise to the highest variability of atrial rate and RR interval, indicating that activation of STAT3 is involved in recovery of atrial and ventricular rhythm. As there are no extrinsic innervation at the embryonic stage investigated and no neurohumoral influence in the culture chamber, the fluctuations of rhythm (dysrhythmias) originated exclusively at the level of the pacemaker tissues, independently of the physiological spontaneous oscillations of heart rate reported previously [45]. These observations and the fact that arrhythmias persisted throughout reoxygenation in $30 \%$ of the AGtreated hearts, strongly suggest that activated STAT3 can protect cardiac automaticity by interacting with pacemaking mechanisms, especially under pathological conditions. We have previously shown that subtle modulation of L-type calcium, $\mathrm{K}_{\mathrm{ATP}}$ and $\mathrm{HCN}$ channels can improve postanoxic recovery of the embryonic heart [6, 44, 46, 54]. It is conceivable that crosstalk between JAK2/STAT3 and RISK pathways may directly or indirectly control finely these ion channels affecting membrane potential, and contributing to protect pacemaker rate under adverse conditions. However, our present findings show clearly that activated STAT3 has no dromo-, ino- and lusitropic effects in the anoxic-reoxygenated embryonic heart since atrioventricular (PR) and intraventricular (QRS widening) conduction, ventricular contractility (shortening) and relaxation (ratio contraction/relaxation velocity) as well as excitation-contraction coupling (EMDv) were not affected by STAT3 inhibition. Additionally, the types of arrhythmias during anoxia and reoxygenation we previously documented [45] were similar in untreated and AG-treated hearts.

ROS-dependent STAT3 $\alpha$ activation during post-anoxic reoxygenation

Our present finding that STAT3 $\alpha$ phosphorylation on tyrosine was ROS-dependent at R10 and R60 is consistent with our preliminary data showing that exogenous $\mathrm{H}_{2} \mathrm{O}_{2}$ also activates STAT3 [Pedretti et al. (personal communication)] and with studies performed in neonatal cardiomyocytes [30] and adult myocardium [33]. However, at R10 there is a strong burst of ROS whereas at R60 ROS production returns to its preanoxic level [44] suggesting that the ROS-dependent mechanisms of STAT3 activation are different during the early (R10) and late (R60) phases of reoxygenation. This phenomenon could be partly due to chemical differences between radical species produced at R10 and R60 (i.e. superoxide anion $\mathrm{O}_{2}^{\circ-}$ being predominantly generated during early reoxygenation) and to variations of the relative contribution of mitochondrial and extramitochondrial (mainly NADPH oxidases) sources of oxyradicals throughout reoxygenation [40]. This issue deserves further investigation. The concept of the ROSmediated protection is also verified in permeabilized cardiac muscle fibers in which the mitochondrial tolerance to anoxia-reoxygenation is improved by $\mathrm{TNF} \alpha$ through ROS production [25].

STAT3 DNA-binding during anoxia-reoxygenation

After its activation STAT3 is known to dimerize and subsequently translocate into the nuclear compartment, where it can modulate expression of specific target genes [20] including iNOS, MnSOD and Cox-2 known to be 
involved in cardioprotection [5, 36, 44]. The facts that STAT3 DNA-binding activity was not altered and that mRNA level of these genes remained stable throughout reoxygenation indicate that translocated STAT3 had no detectable transcriptional activity. Additionally, the level of STAT3 phosphorylation on serine remained constant throughout the experimental protocol $(\sim 2 \mathrm{~h})$, STAT3 requiring phosphorylation on both sites (tyrosine and serine) to be maximally active in the assembly of active transcription complexes [56]. As activated STAT3 was not linked to DNA, we investigated the possible interaction with other signaling pathways, in particular with the RISK pathway.

\section{Crosstalk between JAK2/STAT3 and RISK pathways}

It is still unknown to what extent the survival kinases of the RISK pathway are activated by anoxia-reoxygenation as opposed to ischemia-reperfusion. The temporal profile of phosphorylation shows that components of the RISK pathway (ERK and PI3K-Akt-GSK3beta cascade) were differently modulated during the early (R10) and late (R60) phases of reoxygenation. Phosphorylation of PI3K, Akt and GSK3beta was maximal at R10 whereas activation of ERK2 was delayed at R60 as previously described [12]. The AG490-mediated reduction of phosphorylation of Akt, ERK2 and nuclear GSK3beta indicates clearly that activation of the JAK2/STAT3 pathway can modulate RISK components upon reoxygenation (R10) both in the cytoplasmic (Akt and ERK2) and nuclear (GSK3beta) compartments. We checked the presence of ERK2 in the nuclear compartment in basal conditions whereas ERK2 translocates into the nucleus only when it is phosphorylated. At R10 we found an interaction between ERK2 and STAT3 in homogenate but did not assess specifically the effect of AG490 on ERK2 phosphorylation in nucleus and cytoplasm because ERK2 was not phosphorylated at R10 and consequently not present in the nucleus. The strong basal phosphorylation/inhibition of GSK3beta in the nuclear compartment might be a characteristic of the rapidly growing embryonic ventricle. The additional inhibitory effect of STAT3 on nuclear GSK3beta in the first 10 min of reoxygenation may be determinant as GSK3beta is known to regulate many transcription factors and modulate cellular functions [34]. In $\mathrm{H}_{2} \mathrm{O}_{2}$-treated neonatal [30] and ischemic-reperfused adult $[11,13,15]$ cardiomyocytes STAT3 inhibition reduces also Akt and GSK3beta phosphorylation but the intracellular localization has not yet been established. However, our results show that a preferential and predominant interaction between JAK2/ STAT3 pathway and Akt persists throughout reoxygenation since AG490 leads to strong inactivation of Akt up to R60, which is not the case for GSK3beta, ERK2 and GS.
Whatever the time point investigated, reoxygenationinduced activation of PI3K was unrelated with JAK2/ STAT3 pathway in the embryonic heart like an ischemicreperfused heart model [13], although such a dissociation remains controversial [52]. Furthermore, GS which is a downstream target of GSK3beta, was strongly phosphorylated/inhibited from R10 onward, despite the fact that the phosphorylated form of GSK3beta was inactive. Consequently, other kinases such as PKA, AMPK, CK1 or CK2 [32] may phosphorylate/inhibit GS, reducing glycogen storage which is known to be specially important in the embryonic myocardium and to play a cardioprotective role [41]. The mechanisms by which activation of JAK2/ STAT3 pathway phosphorylate GS at R10 and might transiently reduce glycogen synthesis are beyond the scope of this work. At R10, it appears that there is no dual interaction between PI3K/Akt and JAK2/STAT3 pathways in the embryonic heart. Such an interaction remains controversial in neonatal and adult cardiomyocytes and depends on the type of pathological situation such as ischemic [52] and pharmacological pre- $[15,52]$ and postconditioning [13] and oxidant stress [30]. Regarding

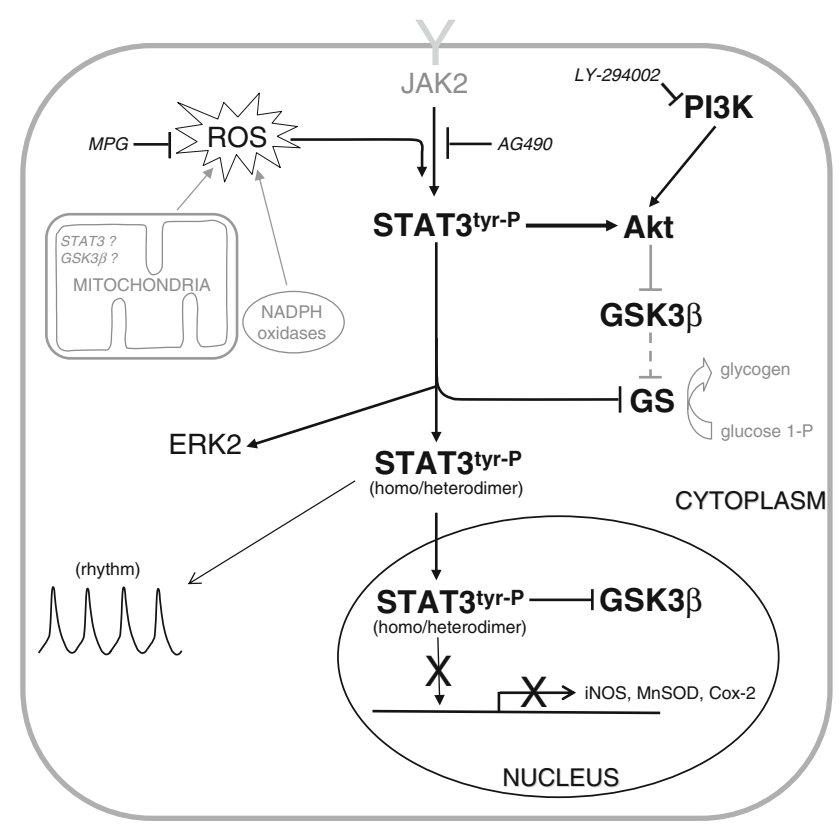

Fig. 10 Schematic representation based on our findings and illustrating STAT3 activation and its possible interaction with the RISK pathway components during the early phase of reoxygenation (10 min) in the embryonic ventricle. In the cytoplasm, mitochondriaand NADPH oxidase-derived ROS stimulate the JAK2/STAT3 pathway which in turn activates Akt and ERK2 and inhibits GS. Activated STAT3 translocates into the nucleus and induces GSK3beta inhibition without binding to DNA. Activation of STAT3 stabilized cardiac rhythm. Arrow, activation; T-shaped symbol, inhibition. Pharmacological agents used are indicated in italics. Dotted lines indicate putative activation/inhibition. Representations in light gray were not explored in the present work 
the importance and the relevance of the RISK pathway, it should be noticed that Skyschally et al. [50] showed that, in hearts of larger mammals like pigs, RISK activation might not be necessary for postconditioning.

In conclusion, this study shows for the first time that the JAK2/STAT3 pathway plays a complex role in the myocardial response to anoxia-reoxygenation during a critical period of cardiogenesis. Indeed, besides its nuclear translocation, the reoxygenation-activated transcription factor STAT3 is also capable of interacting rapidly with various signaling proteins of the RISK pathway present in distinct cellular compartments (Fig. 10). Furthermore, the fact that STAT3 activation improved post-anoxic recovery of cardiac rhythm illustrates the potential role that STAT3 could play in the protection of cardiovascular function in a developing organism. Our findings might be of relevance to better understand the adaptative response of the heart to intermittent, transient or chronic oxygen deprivation during early fetal life, a relatively unexplored area.

Acknowledgments We thank Dr Stéphany Gardier, Dr Noureddine Loukili, Dr Joëlle Rolli and Dr Alexandre Sarre for their advices, Anne-Catherine Thomas for her skillful technical assistance and Dr Antoinette Defaux for providing the histone H1 antibody. This work was supported by the Swiss National Science Foundation, [3100A0-105901].

Conflict of interest None declared.

\section{References}

1. Ananthakrishnan R, Hallam K, Li Q, Ramasamy R (2005) JAKSTAT pathway in cardiac ischemic stress. Vascul Pharmacol 43:353-356. doi:10.1016/j.vph.2005.08.020

2. Boengler K, Heusch G, Schulz R (2010) Mitochondria in postconditioning. Antioxid Redox Signal 2010 Sep 29. [epub ahead of print] doi:10.1089/ars.2010.3309

3. Boengler K, Hilfiker-Kleiner D, Drexler H, Heusch G, Schulz R (2008) The myocardial JAK/STAT pathway: from protection to failure. Pharmacol Ther 120:172-185. doi:10.1016/j.pharmthera. 2008.08.002

4. Boengler K, Hilfiker-Kleiner D, Heusch G, Schulz R (2010) Inhibition of permeability transition pore opening by mitochondrial STAT3 and its role in myocardial ischemia/reperfusion. Basic Res Cardiol 105:771-785. doi:10.1007/s00395-010-0124-1

5. Bolli R, Dawn B, Xuan YT (2003) Role of the JAK-STAT pathway in protection against myocardial ischemia/reperfusion injury. Trends Cardiovasc Med 13:72-79. doi:S105017380 200230X

6. Bruchez P, Sarre A, Kappenberger L, Raddatz E (2008) The L-Type $\mathrm{Ca}+$ and KATP channels may contribute to pacinginduced protection against anoxia-reoxygenation in the embryonic heart model. J Cardiovasc Electrophysiol 19:1196-1202. doi:10.1111/j.1540-8167.2008.01218.x

7. Burton GJ, Jaunaiux E (2001) Maternal vascularisation of the human placenta: does the embryo develop in a hypoxic environment? Gynecol Obstet Fertil 29:503-508. doi:10.1016/ S1297-9589(01)00179-5

8. Chomczynski P, Sacchi N (1987) Single-step method of RNA isolation by acid guanidinium thiocyanate-phenol-chloroform extraction. Anal Biochem 162:156-159. doi:10.1006/abio.1987. 99990003-2697(87)90021-2

9. Dewilde S, Vercelli A, Chiarle R, Poli V (2008) Of alphas and betas: distinct and overlapping functions of STAT3 isoforms. Front Biosci 13:6501-6514. doi:10.2741/3170

10. Foshay K, Rodriguez G, Hoel B, Narayan J, Gallicano GI (2005) JAK2/STAT3 directs cardiomyogenesis within murine embryonic stem cells in vitro. Stem Cells 23:530-543. doi:10.1634/ stemcells.2004-0293

11. Fuglesteg BN, Suleman N, Tiron C, Kanhema T, Lacerda L, Andreasen TV, Sack MN, Jonassen AK, Mjos OD, Opie LH, Lecour S (2008) Signal transducer and activator of transcription 3 is involved in the cardioprotective signalling pathway activated by insulin therapy at reperfusion. Basic Res Cardiol 103:444-453. doi:10.1007/s00395-008-0728-x

12. Gardier S, Pedretti S, Sarre A, Raddatz E (2010) Transient anoxia and oxyradicals induce a region-specific activation of MAPKs in the embryonic heart. Mol Cell Biochem 340:239-247. doi: 10.1007/s11010-010-0423-8

13. Goodman MD, Koch SE, Fuller-Bicer GA, Butler KL (2008) Regulating RISK: a role for JAK-STAT signaling in postconditioning? Am J Physiol Heart Circ Physiol 295:H1649-H1656. doi:10.1152/ajpheart.00692.2008

14. Gough DJ, Corlett A, Schlessinger K, Wegrzyn J, Larner AC, Levy DE (2009) Mitochondrial STAT3 supports Ras-dependent oncogenic transformation. Science 324:1713-1716. doi:10.1126/ science. 1171721

15. Gross ER, Hsu AK, Gross GJ (2006) The JAK/STAT pathway is essential for opioid-induced cardioprotection: JAK2 as a mediator of STAT3, Akt, and GSK-3 beta. Am J Physiol Heart Circ Physiol 291:H827-H834. doi:10.1152/ajpheart.00003.2006

16. Hamburger V, Hamilton $H$ (1951) A series of normal stages in the development of the chick embryo. J Morphol 88:49-92. doi: 10.1002/aja.1001950404

17. Hausenloy DJ, Lecour S, Yellon DM (2010) RISK and SAFE pro-survival signalling pathways in ischaemic postconditioning: two sides of the same coin. Antioxid Redox Signal 2010 Oct 26 [epub ahead of print]. doi:10.1089/ars.2010.3360

18. Hausenloy DJ, Yellon DM (2006) Survival kinases in ischemic preconditioning and postconditioning. Cardiovasc Res 70:240-253. doi:10.1016/j.cardiores.2006.01.017

19. Heusch G, Boengler K, Schulz R (2008) Cardioprotection: nitric oxide, protein kinases, and mitochondria. Circulation 118:19151919. doi:10.1161/CIRCULATIONAHA. 108.805242

20. Imada K, Leonard WJ (2000) The Jak-STAT pathway. Mol Immunol 37:1-11. doi:S0161589000000183

21. Jensen A, Garnier Y, Berger R (1999) Dynamics of fetal circulatory responses to hypoxia and asphyxia. Eur J Obstet Gynecol Reprod Biol 84:155-172. doi:S030121159800325X

22. Kelly RF, Lamont KT, Somers S, Hacking D, Lacerda L, Thomas P, Opie LH, Lecour S (2010) Ethanolamine is a novel STAT-3 dependent cardioprotective agent. Basic Res Cardiol 105:763770. doi:10.1007/s00395-010-0125-0

23. Ko ML, Shi L, Grushin K, Nigussie F, Ko GY (2010) Circadian profiles in the embryonic chick heart: L-type voltage-gated calcium channels and signaling pathways. Chronobiol Int 27:1673-1696. doi:10.3109/07420528.2010.514631

24. Kurdi M, Booz GW (2007) Can the protective actions of JAKSTAT in the heart be exploited therapeutically? Parsing the regulation of interleukin-6-type cytokine signaling. J Cardiovasc Pharmacol 50:126-141. doi:10.1097/FJC.0b013e318068dd49 00005344-200708000-00005 
25. Lacerda L, McCarthy J, Mungly SF, Lynn EG, Sack MN, Opie LH, Lecour S (2010) TNFalpha protects cardiac mitochondria independently of its cell surface receptors. Basic Res Cardiol 105:751-762. doi:10.1007/s00395-010-0113-4

26. Lacerda L, Somers S, Opie LH, Lecour S (2009) Ischemic postconditioning protects against reperfusion injury via the SAFE pathway. Cardiovasc Res 84:201-208. doi:10.1093/cvr/cvp274

27. Levrand S, Pesse B, Feihl F, Waeber B, Pacher P, Rolli J, Schaller MD, Liaudet L (2005) Peroxynitrite is a potent inhibitor of NF-\{kappa $\} \mathrm{B}$ activation triggered by inflammatory stimuli in cardiac and endothelial cell lines. J Biol Chem 280:34878-34887. doi:10.1074/jbc.M501977200

28. Lim CP, Cao X (2006) Structure, function, and regulation of STAT proteins. Mol Biosyst 2:536-550. doi:10.1039/b606246f

29. Livak KJ, Schmittgen TD (2001) Analysis of relative gene expression data using real-time quantitative PCR and the 2(-Delta Delta C(T)) Method. Methods 25:402-408. doi:10.1006/meth. 2001.1262S1046-2023(01)91262-9

30. Lu Y, Zhou J, Xu C, Lin H, Xiao J, Wang Z, Yang B (2008) JAK/ STAT and PI3 K/AKT pathways form a mutual transactivation loop and afford resistance to oxidative stress-induced apoptosis in cardiomyocytes. Cell Physiol Biochem 21:305-314. doi: $10.1159 / 000129389$

31. Maltepe E, Simon MC (1998) Oxygen, genes, and development: an analysis of the role of hypoxic gene regulation during murine vascular development. J Mol Med 76:391-401. doi: $10.1007 / \mathrm{s} 001090050231$

32. Marks F, Klingmüller U, Müller-Decker K (2009) Cellular signal processing. Garland Science, New York, pp 194-196

33. McCormick J, Barry SP, Sivarajah A, Stefanutti G, Townsend PA, Lawrence KM, Eaton S, Knight RA, Thiemermann C, Latchman DS, Stephanou A (2006) Free radical scavenging inhibits STAT phosphorylation following in vivo ischemia/ reperfusion injury. Faseb J 20:2115-2117. doi:10.1096/fj.066188fje

34. Meares GP, Jope RS (2007) Resolution of the nuclear localization mechanism of glycogen synthase kinase-3: functional effects in apoptosis. J Biol Chem 282:16989-17001. doi:10.1074/jbc. M700610200

35. Miyamoto S, Rubio M, Sussman MA (2009) Nuclear and mitochondrial signalling Akts in cardiomyocytes. Cardiovasc Res 82:272-285. doi:10.1093/cvr/cvp087

36. Negoro S, Kunisada K, Fujio Y, Funamoto M, Darville MI, Eizirik DL, Osugi T, Izumi M, Oshima Y, Nakaoka Y, Hirota H, Kishimoto T, Yamauchi-Takihara K (2001) Activation of signal transducer and activator of transcription 3 protects cardiomyocytes from hypoxia/reoxygenation-induced oxidative stress through the upregulation of manganese superoxide dismutase. Circulation 104:979-981. doi:10.1161/hc3401.095947

37. Negoro S, Kunisada K, Tone E, Funamoto M, Oh H, Kishimoto T, Yamauchi-Takihara K (2000) Activation of JAK/STAT pathway transduces cytoprotective signal in rat acute myocardial infarction. Cardiovasc Res 47:797-805. doi:S0008-6363(00) 00138-3

38. Pantos C, Xinaris C, Mourouzis I, Malliopoulou V, Kardami E, Cokkinos DV (2007) Thyroid hormone changes cardiomyocyte shape and geometry via ERK signaling pathway: potential therapeutic implications in reversing cardiac remodeling? Mol Cell Biochem 297:65-72. doi:10.1007/s11010-006-9323-3

39. Raddatz E, Gardier S, Sarre A (2006) Physiopathology of the embryonic heart (with special emphasis on hypoxia and reoxygenation). Ann Cardiol Angeiol (Paris) 55:79-89. doi:10.1016/ j.ancard.2006.02.007

40. Raddatz E, Thomas AC, Sarre A, Benathan M (2010) Differential contribution of mitochondria, NADPH-oxidases and glycolysis to region-specific oxidant stress in the anoxic-reoxygenated embryonic heart. Am J Physiol Heart Circ Physiol 2010 Dec 30 [epub ahead of print]. doi:10.1152/ajpheart.00827.2010

41. Romano R, Rochat AC, Kucera P, De Ribaupierre Y, Raddatz E (2001) Oxidative and glycogenolytic capacities within the developing chick heart. Pediatr Res 49:363-372. doi:0031-3998/ 01/4903-0363

42. Rubio M, Avitabile D, Fischer K, Emmanuel G, Gude N, Miyamoto S, Mishra S, Schaefer EM, Brown JH, Sussman MA (2009) Cardioprotective stimuli mediate phosphoinositide 3-kinase and phosphoinositide dependent kinase 1 nuclear accumulation in cardiomyocytes. J Mol Cell Cardiol 47:96-103. doi: 10.1016/j.yjmcc.2009.02.022

43. Sarre A, Gardier S, Maurer F, Bonny C, Raddatz E (2008) Modulation of the c-Jun N-terminal kinase activity in the embryonic heart in response to anoxia-reoxygenation: involvement of the $\mathrm{Ca} 2+$ and mitoKATP channels. Mol Cell Biochem 313:133-138. doi:10.1007/s11010-008-9750-4

44. Sarre A, Lange N, Kucera P, Raddatz E (2005) mitoKATP channel activation in the postanoxic developing heart protects E-C coupling via NO-, ROS-, and PKC-dependent pathways. Am J Physiol Heart Circ Physiol 288:H1611-H1619. doi:10.1152/ ajpheart.00942.2004

45. Sarre A, Maury P, Kucera P, Kappenberger L, Raddatz E (2006) Arrhythmogenesis in the developing heart during anoxia-reoxygenation and hypothermia-rewarming: an in vitro model. J Cardiovasc Electrophysiol 17:1350-1359. doi:10.1111/j.1540-8167. 2006.00637.x

46. Sarre A, Pedretti S, Gardier S, Raddatz E (2010) Specific inhibition of HCN channels slows rhythm differently in atria, ventricle and outflow tract and stabilizes conduction in the anoxic-reoxygenated embryonic heart model. Pharmacol Res 61:85-91. doi:10.1016/ j.phrs.2009.09.007

47. Schaefer TS, Sanders LK, Park OK, Nathans D (1997) Functional differences between Stat3alpha and Stat3beta. Mol Cell Biol 17:5307-5316. doi:0270-7306/97/\$04.0010

48. Sedmera D, Kucera P, Raddatz E (2002) Developmental changes in cardiac recovery from anoxia-reoxygenation. Am J Physiol Regul Integr Comp Physiol 283:R379-R388. doi:10.1152/ajpregu. 00534.2001

49. Sedmera D, Pexieder T, Vuillemin M, Thompson RP, Anderson RH (2000) Developmental patterning of the myocardium. Anat Rec 258:319-337. doi:10.1002/(SICI)1097-0185(20000401) 258:4<319:AID-AR1>3.0.CO;2-O

50. Skyschally A, van Caster P, Boengler K, Gres P, Musiolik J, Schilawa D, Schulz R, Heusch G (2009) Ischemic postconditioning in pigs: no causal role for RISK activation. Circ Res 104:15-18. doi:10.1161/CIRCRESAHA.108.186429

51. Stephanou A, Brar BK, Knight RA, Latchman DS (2000) Opposing actions of STAT-1 and STAT- 3 on the Bcl-2 and Bcl-x promoters. Cell Death Differ 7:329-330. doi:10.1038/sj.cdd. 4400656

52. Suleman N, Somers S, Smith R, Opie LH, Lecour S (2008) Dual activation of STAT-3 and Akt is required during the trigger phase of ischaemic preconditioning. Cardiovasc Res 79:127-133. doi: $10.1093 / \mathrm{cvr} / \mathrm{cvn} 067$

53. Takeda K, Noguchi K, Shi W, Tanaka T, Matsumoto M, Yoshida N, Kishimoto T, Akira S (1997) Targeted disruption of the mouse Stat3 gene leads to early embryonic lethality. Proc Natl Acad Sci USA 94:3801-3804. doi:0027-8424y97y943801-4\$2.00y0

54. Tenthorey D, de Ribaupierre Y, Kucera P, Raddatz E (1998) Effects of verapamil and ryanodine on activity of the embryonic chick heart during anoxia and reoxygenation. J Cardiovasc Pharmacol 31:195-202. doi:00005344-199802000-00004

55. Wegrzyn J, Potla R, Chwae YJ, Sepuri NB, Zhang Q, Koeck T, Derecka M, Szczepanek K, Szelag M, Gornicka A, Moh A, Moghaddas S, Chen Q, Bobbili S, Cichy J, Dulak J, Baker DP, 
Wolfman A, Stuehr D, Hassan MO, Fu XY, Avadhani N, Drake JI, Fawcett P, Lesnefsky EJ, Larner AC (2009) Function of mitochondrial Stat3 in cellular respiration. Science 323:793-797. doi:10.1126/science. 1164551
56. Wen Z, Zhong Z, Darnell JE Jr (1995) Maximal activation of transcription by Stat 1 and Stat 3 requires both tyrosine and serine phosphorylation. Cell 82:241-250. doi:0092-8674(95)90311-9 